\title{
Stochastic dynamic programming model for optimal resource allocation in vehicular ad hoc networks
}

\author{
M BHUVANESWARI $^{1, *}$, B PARAMASIVAN ${ }^{1}$ and A KANDASAMY ${ }^{2}$ \\ ${ }^{1}$ Department of Computer Science and Engineering, National Engineering College, Kovilpatti 628503, India \\ ${ }^{2}$ Department of Mathematical and Computational Sciences, National Institute of Technology Karnataka, \\ Mangalore 575025, India \\ e-mail: itsbhuvana@gmail.com
}

MS received 28 January 2017; revised 4 July 2017; accepted 4 September 2017; published online 11 April 2018

\begin{abstract}
Vehicular ad hoc network (VANET) is an emerging trend where vehicles communicate with each other and possibly with a roadside unit to assist various applications like monitoring, managing and optimizing the transportation system. Collaboration among vehicles is significant in VANET. Resource constraint is one of the great challenges of VANETs. Because of the absence of centralized management, there is pitfall in optimal resource allocation, which leads to ineffective routing. Effective reliable routing is quite essential to achieve intelligent transportation. Stochastic dynamic programming is currently employed as a tool to analyse, develop and solve network resource constraint and allocation issues of resources in VANET. We have considered this work as a geographical-angular-zone-based two-phase dynamic resource allocation problem with a homogeneous resource class. This work uses a stochastic dynamic programming algorithm based on relaxed approximation to generate optimal resource allocation strategies over time in response to past task completion status history. The second phase resource allocation uses the observed outcome of the first phase task completion to provide optimal viability in resulting decisions. The proposed work will be further extended for the scenario that deals with heterogeneous resource class. Simulation results show that the proposed scheme works significantly well for the problems with identical resources.
\end{abstract}

Keywords. Stochastic dynamic programming; relaxed formulation; vehicular ad hoc networks; resource allocation.

\section{Introduction}

Vehicular ad hoc networks (VANETs) are self-organizing and are formed directly by a set of smart vehicles. They can interact without using fixed infrastructure or centralized administration. In recent years there is a sharp increase of vehicles on roads. This attracts increasing attention of leading car manufacturers who are willing to jointly work with national government agencies and wireless network research groups to develop intelligent transportation systems [1]. This scenario causes VANET as an emerging research area in wireless network community. The characteristics of VANETs pose great challenges in the routing and resource allocation protocol design because of their dynamic network topologies, lack of centralized control and self-organizing character. Collaboration and scheduling of resources are the most conscious points for efficient routing. Successful packet forwarding (PF) from source to destination is the

*For correspondence underlying principle of critical applications. Such communication can be named as a task.

Optimal resource allocation and utilization plays a vital role in reliable VANET communication. Node mobility creates more opportunities for frequent resource handover in VANET; because of high dynamics in network topology, collaboration between nodes is more challenging in VANETs. Therefore it is essential to develop an effective resource allocation scheme to achieve time-sensitive communication from source to destination in a reliable manner. In general, unbalanced resource allocation and utilization does not contribute in tasks completion, which indirectly degrades the network performance. Existing schemes are not suitable for enhancing VANET resource allocations because of routing overhead. Since the vehicles move in one direction in a particular lane, there is no need to consider the neighbour nodes, which are placed in $360^{\circ}$. To increase the network performance, the network scenario was analysed in terms of available resources, type of resources, past history of the communication over the resource allocation and probable number of future 
communication. Dynamic programming approaches [2] provide effective solutions to resource allocation issues in VANETs. They apply to a variety of resource allocation applications, namely fleet management, medicinebased personnel appointment scheduling and economics where the resources are in different forms like vehicles, containers, doctors, nurses, cash and stocks. Similarly, the uncertainty may have different characterizations in these applications. An approximate stochastic dynamic programming (SDP) [3] allows nodes with a number of possible actions with clear strategies for devising an effective decision on optimal resource allocation.

Though the VANET research community has focussed on many challenges in VANETs, very few research work has progressed on resource allocation issues. Among those efforts, herewith we have analysed a few resource allocation techniques for vehicular networks. Rawat et al [4] proposed a dynamic spectrum access scheme for intravehicle communication. In their work, they assumed that all vehicles are equipped with a software-defined RF transceiver. They considered the allocation of only radio transceivers that lie in the transmission range. Bejaoui [5] presented a throughput-assured cross-layered control algorithm that makes use of a priority function to evolve a fair resource scheduling decision. Significant VANET parameters to take the decision were also considered. GTBA does not take the resource types and availability into consideration. It only bothered to attain throughput service and not other services. Xing et al [6] devised a resource allocation technique based on dynamic programming for vehicle to vehicle communication. They grouped the vehicles into multiple clusters. Each cluster member utilizes a single cellular user's resource for communication. For this the authors considered all vehicles for clustering. The control packets were flooded around $360^{\circ}$ of an elected cluster head. Such a situation leads to higher routing overhead. This scheme does not amply address the incomplete tasks over the single-phase resource allocation.

We propose a protocol, two stages approximate SDP algorithm, to expand the set of allowable resource allocation strategies on admissible decisions for enhancing routing of VANETs. Differently from the afore-mentioned techniques, this method reveals the best outcomes and it solves the resource allocation problem with nearly linear computational complexity with a higher number of tasks and resources.

The remainder of the paper contains six sections. The related work is summarized in section 2, which highlights the differences of existing techniques and our proposed work. Section 3 describes our SDP based on two-phase approximation for resource allocation. Section 4 presents the environmental set-up of the proposed work's simulation. It also shows the results and a relevant performance analysis. Finally, section 5 presents the conclusions and scope for future direction.

\section{Related works}

In VANETs applications, one is often interested in maximizing the performance of the communication application while operating within constraints on resources. Effective data transmission in VANETs is a challenge for various applications. Araniti et al [7] investigated and reported the usability of long-term evolution (LTE) for VANET applications. They also analysed the strength and weakness of LTE standardization in terms of VANET applications. Krishnamurthy [8] addressed various research approaches in applying hidden Markov model to optimal scheduling of sensors. Several such approaches have been developed, such as binary programming for sensor scheduling [9], scheduling of wake-up duration to reduce a sensor network energy consumption using dynamic programming [10], analysing the sensor resource allocation for tracking using outer approximation [11], spectrum sharing scheme between cellular users and ad hoc device-to-device users [12] and cross-layer communication in vehicle-to-infrastructure multi-hop communication of vehicular networks [13]. Williams et al [14] presented approximate dynamic programming systems to a communication-constrained sensor management problem. Lim et al [15] proposed an adaptive approach for scheduling Medium Access Control layer of VANET. It aims at minimizing average delay and maximizing packet success rate. An adaptive joint optimization with threshold structure dynamic programming has been used as a closed-loop feedback control system. It is designed to optimize contention window size and transmission power. This work does not consider heterogeneous classes of resources. Cheung et al [16] studied random access in a drive-through scenario. The roadside access points (APs) provide temporary Internet access for vehicles. They find the optimal transmission policy with a single AP on random vehicular traffic arrivals. This problem is casted as a finite horizon sequential decision problem, solved using dynamic programming. Various conditions were derived under which the optimal transmission policy has a threshold structure. A monotone DORA algorithm has been proposed, which has lower computational complexity for this drive-through scenario. The optimal transmission policy for multiple APs has not been considered. Dhurandher et al [17] introduced an efficient routing protocol. It finds the minimum possible path length between a source and a destination. It involves minimum nodes in data transmission. A novel angular position algorithm is exploited to select the most suitable node for transmission. It is useful to achieve proper network connectivity among nodes with minimum power consumption. This work considers only energy conservation. It is not concentrated on optimal resource allocation. Paramasivan et al [18] introduced a VANET routing protocol by combining link state information, connection lifetime prediction and store-stay-spray mechanism. It uses geographical-angular-region-based routing to choose the most suitable next hop for PF. The 
angular region consideration gives proper network connectivity among nodes with minimum energy consumption. An ensemble learning algorithm is used to predict the link state information and connection lifetime. This information gives information to every node in taking decision on the best path selection. Considerable reliability in routing is also achieved through DTN concepts of store-stay-spray. Vakilinia et al [19] proposed a model for cloud resources in profiles of mobile application. Wireless interfaces and cloud resources are allocated using an event-based finite state model and dynamic constraint programming method. This proposed model is based on the wireless network cloud concept. A multi-objective optimization approach is used to determine the appropriate process power, transmission power, QoS profiles and cloud off-loading. There is no chance for mobility in cloud environment. Castanon and Wohletz [1] considered a class of stochastic resource allocation problems. The SDP is used to solve this problem. The approximation is also used in the model-predictive control algorithm by overcoming the demerits of SDP. It takes optimal resource allocation decisions by considering previous stage task completion history. They extended the work with relaxed formulation and model-predictive control approach for heterogeneous resources. The modelpredictive control algorithm based on relaxed SDP can solve the relaxed problem in a time that is nearly linear with large number of tasks. Multiple assignments of simultaneous resources have not been considered in this work. Moreover, the characteristics of wireless mobile networks are not considered. Our work is partially close to that of Dhurandher et al [17] and Castanon and Wohletz [1]. Compared with related work [7] to [1], our work is distinguished by type and characteristics of network resources. It is also categorized with the existing work by geographical-angular-zone-based resources allocation for efficient routing. It has been formulated as an approximate SDP algorithm to seek the best resource allocation outcome. The goal of this scheme is to find an optimal solution for efficient routing by allocating the available resources effectively. It also tries to solve the same with computational complexity that grows linearly with the number of tasks and resources. As an outcome, we derive different analyses of node strategies to seek the best outcome of the game. As a new effort, this is the first attempt to employ relaxed approximation SDP algorithm that works on geographical angular zone to generate optimal resource allocation strategies for VANETs.

\section{Relaxed approximation SDP model in VANETs}

The proposed work assumes this resource allocation approach as a two-phase problem. Consider that there are ' $V_{n}$ ' number of vehicles and ' $D_{T n}$ ' number of data transmissions. Assume that all vehicles are equipped with homogeneous resource class. Specifically, each OBU has the same configuration of sensors, communication and control subsystems. It also equips with the same battery power. Hence, there is a total of ' $R_{T n}$ ' homogeneous resource class. In VANET, since vehicles move in a particular lane towards a single direction, there is no need for considering the total resources, which is placed in $360^{\circ}$ for forwarding the packets. Figure 1 shows the selection of optimal angular zone for data transmission. If a vehicle ' $V_{i}$ ' wants to send a data packet to ' $V_{j}$ ', ' $V_{i}$ ' calculates angular transmission zone by joining the ' $V_{i}$ ' with ' $V_{j}$ '. It is represented as vector $\left(r V_{i} V_{j}\right)$. In this zone, $R_{o}$ is an optimal number of resources considered for resource allocation for packet transmission from $V_{i}$ to $V_{j}$. The heed zone is another zone that holds the angle $\left(\theta_{4}, \theta_{5}\right) . R_{h}$ is the number of resources belonging to this region. This can also be considered for packet transmission. The resources in futile region need not be considered for data transmission. Specifically, this approach considers only a minimum number of resources (especially $R_{o}$, for some worst case $R_{h}$ may also be included) for data transmission. Hence, $R_{n}$ is the optimal number of resources $<R_{n} \mid R_{n} \leq R_{o}+R_{h}$ and $R_{n} \in R_{T n}>$ that can be assigned to each communication over two possible phases. Based on the completion level, each data transmission was initialized with a communication $\operatorname{cost} C_{c}$. Each resource is associated with its own cost called $C_{R}$. When a resource is assigned to a data transmission $D_{T i}$ in phase $k, P\left(D_{T i}(k)\right)$ is the probability that the resource completes the data transmission $D_{T i}$ successfully in the $k$ th phase. The number of resources assigned to a particular data transmission $D_{T i}$ at phase 1 is represented as $A_{D_{T i}}(1) . P\left(D_{T i}(1)\right)$ is the probability that the resource completes the data transmission $D_{T i}$ successfully in the 1st phase.

$$
P_{F}\left(D_{T i}, 1\right)=\left(1-P\left(D_{T i}(1)\right)\right)^{A_{i}(1)}
$$

Equation (1) represents the probability that the data transmission $D_{T i}$ is not completed by the resource allocation

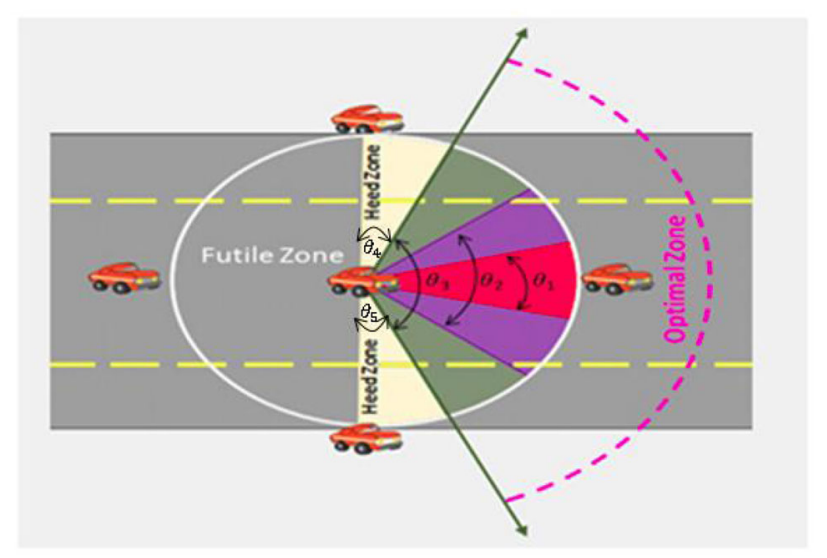

Figure 1. Process of separating angular zone for optimal data transmission. 


\begin{tabular}{|c|c|c|c|c|c|c|}
\hline \multirow{2}{*}{$w^{\prime}(0)$} & $\mathbf{D}_{\mathrm{T} 1}$ & $\mathrm{D}_{\mathrm{T} 2}$ & $\mathbf{D}_{\mathrm{T3}}$ & $\ldots \ldots \ldots \ldots \ldots$ & $D_{\text {Tn-1 }}$ & $\mathrm{D}_{\text {Tn }}$ \\
\hline & 1 & 1 & 1 & $\ldots \ldots \ldots \ldots \ldots$ & 1 & 1 \\
\hline \multirow{2}{*}{$w^{\prime} \mid A^{\prime}(1)$} & $\mathrm{D}_{\mathrm{T1}}$ & $D_{T 2}$ & $\mathbf{D}_{\mathrm{T3}}$ & $\ldots \ldots \ldots \ldots \ldots$ & $D_{T n-1}$ & $D_{\text {Tn }}$ \\
\hline & 0 & 0 & 1 & $\ldots \ldots \ldots \ldots \ldots$ & 1 & 0 \\
\hline \multirow{2}{*}{$w^{\prime} \mid A^{\prime}(2)$} & $\mathrm{D}_{\mathrm{T} 1}$ & $\mathrm{D}_{\mathrm{T} 2}$ & $\mathbf{D}_{\mathrm{T} 3}$ & $\ldots \ldots \ldots \ldots \ldots$ & $D_{\text {Tn-1 }}$ & $\mathrm{D}_{\text {Tn }}$ \\
\hline & 0 & 0 & 0 & $\ldots \ldots \ldots \ldots \ldots$ & 0 & 0 \\
\hline
\end{tabular}

Figure 2. Evolution of the routing state vector.

$A_{D_{T i}}(1)$. At each phase end, the uncompleted data transmission will be noted and represented as routing state vector. It is a binary vector set that holds two possible values 0 or 1 . It is formally defined as $w=\{0,1\}^{R_{n}}$. Suppose that $w D_{T i}=0$ means the $i$ th data transmission was completed in phase 1 whereas the $i$ th data transmission was not completed in phase 1 resource allocation can be represented as $w D_{T i}=1$. $P\left(w^{\prime} \mid A^{\prime}(1)\right)$ is a probability distribution on possible outcome where $A^{\prime}(1)$ is a vector of phase 1 resource allocations. The resource allocations in phase 2 depends on the outcome $w^{\prime}(1)$, which can be observed as $A^{\prime}\left(2, w^{\prime}\right)$. They are called as resource strategies for phase 2 . Figure 2 presents an evolution of the routing state across phases. The evolution of the routing state vector starts from the derivation of $w^{\prime}(0)$. It consists of all ones, which indicate that no data transmissions are complete initially. The routing state transits from $w^{\prime}(0)$ to $w^{\prime}(1)$ by the resource allocation $A^{\prime}(1)$. This state transition also depends on the probability $P_{F}\left(D_{T i}, 1\right)$ that produces $w D_{T i}=1$. The state transition is illustrated in figure 3 . The resource strategy $A^{\prime}\left(2, w^{\prime}(1)\right)$ leads the state transition to $w^{\prime}(2)$. The probability that data transmission $_{i}$ is not successfully performed in either phase 1 or phase 2 is computed as follows:

$$
P_{F}\left(D_{T i}, 2\right)=\sum_{A^{\prime}} P\left(w^{\prime}(1) \mid A^{\prime}(1)\right) \times F_{1}^{\prime} \times F_{2}^{\prime}
$$

where

$$
F_{1}{ }^{\prime}=I\left(w D_{T i}=1\right)
$$

$I$ is an index function and

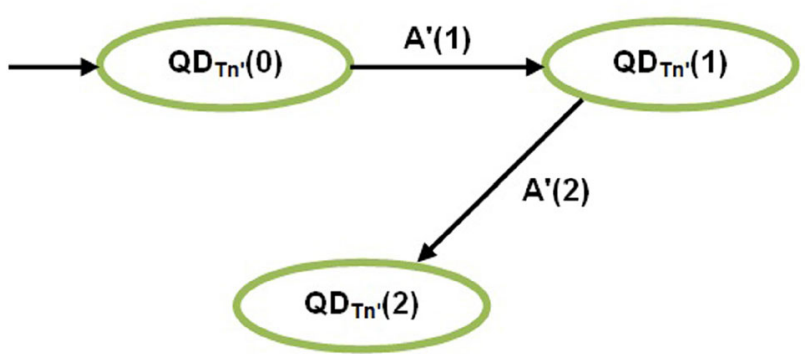

Figure 3. State transition of the routing state vector.

$$
\begin{aligned}
& F_{2}{ }^{\prime}=\left(1-P_{D_{T i}}(2)\right)^{A_{D_{T i}}(2, w(1))} \\
& Z=P\left(w^{\prime}(1) \mid A^{\prime}(1)\right)=\prod_{i \mid w_{i}=0}\left[P\left(D_{T i}(1)\right)\right]
\end{aligned}
$$

The goal of optimal control problem is to minimize the expected cost of using resources and expected incomplete data transmission value by selecting the resource allocations $A^{\prime}(1)$ and resource strategies $A^{\prime}\left(2, w^{\prime}(1)\right)$. This can be calculated using Eqs. (4) and (5) along with the constraints given in Eq. (6):

$$
\begin{gathered}
E_{c}=\sum_{i=1}^{D_{T n}} C_{c i} . I\left(W_{i}(2)=1\right)+C_{R} \sum_{i=1}^{D_{T n}} A_{D_{T i}}(1)+A_{D_{T i}}\left(2, w^{\prime}(1)\right) \\
E_{c}=\sum_{i=1}^{D_{T n}}\left[C_{c i} . P\left(D_{T i}, 2\right)+C_{R} \sum_{w \prime} Z \times\left(A_{D_{T i}}(1)\right.\right. \\
\left.\left.+A_{D_{T i}}\left(2, w^{\prime}(1)\right)\right)\right] \\
\\
\sum_{i=1}^{D_{T n}}\left(A_{D_{T i}}(1)+A_{D_{T i}}\left(2, w^{\prime}(1)\right)\right) \leq R_{n}
\end{gathered}
$$

Resource allocation for VANET routing is a two-phase stochastic control problem where the discrete state space and action space grow exponentially with respect to the number of data transmissions. In this part, we consider this problem from the second phase. Assume that there are $D_{T i}{ }^{\prime}$ number of incomplete tasks in the first phase $w^{\prime}(1)$ and $R_{n}{ }^{\prime}$ number of remaining resources. In this scenario, the second phase of the resource allocation problem can be described as follows:

$$
\sum_{i=1}^{D_{T i^{\prime}}} C_{c j}\left(1-P_{j}\right)^{A_{D_{T j}}}+C_{R} A_{D_{T j}} .
$$

This equation is subject to the constraint

$$
\sum_{j=1}^{D_{T_{i}^{\prime}}} x_{j} \leq R_{n}{ }^{\prime} .
$$

This approach is the fastest one for optimal resource allocation $A_{2}\left(D_{T i}{ }^{\prime}, w^{\prime}\right)$ and the optimal value is $R A_{2}\left(w^{\prime}, R_{n}{ }^{\prime}\right)$. The key structural outcome is stated by the following lemma.

Lemma 1 As per the definition of second phase problem in Eqs. (7) and (8), resource allocation can be described as follows. In the following equation, where $\delta$ is a non-negative real value of the slopes of linear functions $f_{i}()$

$$
A_{j}^{+} \in_{A_{j} \in\left[0, R_{n}^{\prime}\right]}^{\min }\left[f_{i}\left(A_{j}\right)+\delta^{+} A_{j}\right]
$$

Proof The existence of $\delta$ is ensured by the convexity of single constraint stated in Eq. (8). The solution for Eq. (9) corresponds to negative values of the slopes of $f_{i}()$, where 
$i=1, \ldots, D_{T i}{ }^{\prime}$. Hence, the values of $\delta$ can be limited to the discrete set of integer values. The optimal allocation of remaining resources $R_{n}{ }^{\prime}$ monotonically increases for each coordinate of function $f_{i}()$. This property is used to solve the second phase resource allocation process with the complexity of $\mathrm{O}\left(D_{T i}+R_{n}{ }^{\prime} \ln \left(D_{T i}+R_{n}{ }^{\prime}\right)\right)$. The SDP is a fast procedure for finding the optimal resource allocation $\left(A_{j}^{+}\right)$strategies $A_{2}\left(D_{T i}, w^{\prime}(1)\right)$; expected resource allocation cost function (for the second phase of the resource allocation problem) can be defined as $E_{2}^{+}\left(w^{\prime}(1), R_{n}{ }^{\prime}\right)$. This cost function can be calculated by considering the first phase possible resource allocation result $w^{\prime}(1)$ and remaining unutilized optimal zone resources $\left(R_{n}{ }^{\prime}\right)$. At this point, we consider the first phase of this resource allocation problem. The following SDP function can be satisfied by the solution of Eqs. (4) and (6):

$$
E^{+}=\min _{A^{\prime}(1) \in\left\{0, \ldots, R_{n}\right\}} \sum_{w^{\prime}} Z \times E_{2}^{+}\left(w^{\prime}(1), R_{n}-Y+C_{R} \times Y\right.
$$

where the value of $Y=\sum_{D_{T 1}}^{D_{T n}} A_{i}(1) \leq R_{n}$. The value for $P\left(w^{\prime} \mid A^{\prime}(1)\right)$ is already defined by Eq. (3). This algorithm can also be solved by the approximate greedy optimization approach illustrated in Algorithm 1.

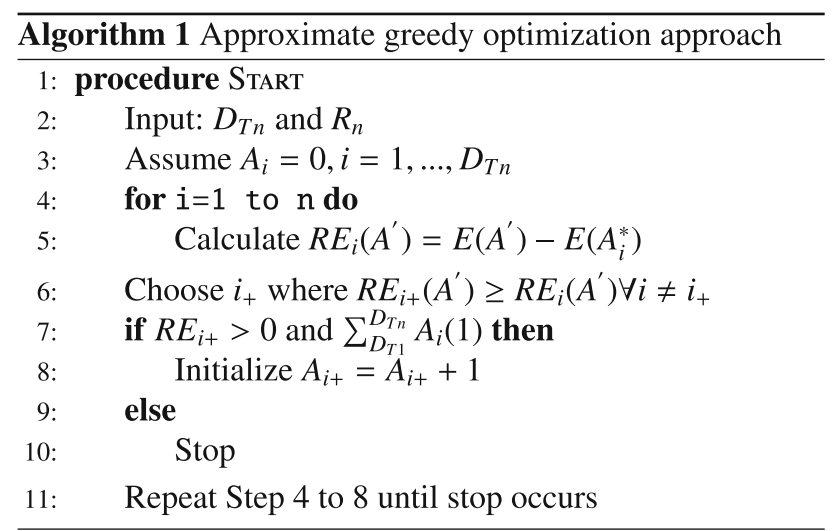

\section{Performance analysis}

The proposed optimal two-phase resource allocation approach has been implemented in VanetMobiSim and Network Simulator (NS3). Efficient Routing was the main objective of this proposed work simulation. Nodes followed the traffic pattern generated by VANET Simulator (VanetMobiSim). We have extracted and used the Madurai (Tamilnadu) map from OpenStreetMap for our simulation. The detailed simulation parameters' configuration is presented in table 1.

IEEE 802.11p PHY parameters set-up is carried out in the WirelessPhyExt configuration. The parameters are CSThresh, Energy Detection Threshold and CCAModel
Table 1. Simulation parameters configuration.

\begin{tabular}{lc}
\hline Simulation parameters & Configuration values \\
\hline Simulation area & $1500 \mathrm{~m} \times 1500 \mathrm{~m}$ \\
No. of OBUs used & 100 \\
No. of RSUs deployed & 10 \\
RSUs transmission range & $200 \mathrm{~m}$ \\
OBUs transmission range & $50 \mathrm{~m}$ \\
Network interface type & PhyWirelessPhyExt \\
MAC type & IEEE $802.11 \mathrm{p} \mathrm{MacExt}$ \\
Radio propagation model & Nakagami-m $(m=3)$ \\
Packet size & 1024 bytes \\
Network traffic & CBR $(1024$ bytess, rate: 2$)$ \\
Vehicle speed & $30-80 \mathrm{~km} / \mathrm{h}$ \\
\hline
\end{tabular}

Threshold, Frequency, Noise, Radio circuit gain/loss rate, Owner Monitor Threshold, Basic Modulation Scheme and so on. IEEE $802.11 \mathrm{p}$ MAC parameters configuration is carried out in the $802.11 \mathrm{pExt}$ set-up. The parameters are CWMin, CWMax, Short Inter-frame Space (SIFS), Slottime, Short Retry Limit, Long Retry Limit, RTS Threshold and so on. The geographical-angular-zone-based relaxed approximation stochastic dynamic programming (GARASDP) model analysed the impact of two-phase resource allocation. It also evaluated the outcome of first phase's resource allocation strategies. We conducted several sets of experiments with homogeneous resources to calculate the effectiveness of GARA-SDP approach. We initialized 10 15 data transmissions. Each data transmission takes a random value in the space of 5-15. The success probabilities of each task carry a value in the interval $\{0.8,1.0\}$ in a random fashion. This experiment assigns 8-17 numbers of resources for completing each data transmission. Each data transmission includes 20-30 numbers of PF and obtains results as expected value of uncompleted data transmission using Heuristic Resource allocation algorithm (HRAA) by the Incremental Dynamic Programming approach (IDP). In table 2, the performance of GARA-SDP is compared to those of HRAA and IDP. The results show that the performance of GARA-SDP is optimal; the reason for this better performance is that GARA-SDP uses the

Table 2. Performance on average expected value of uncompleted data transmission.

\begin{tabular}{lrrccc}
\hline$(1)$ & $(2)$ & $(3)$ & GARA-SDP (\%) & HRAA (\%) & IDP (\%) \\
\hline 11 & 28 & 13 & 0.13 & 0.624 & 0.24 \\
10 & 22 & 8 & 0 & 0.56 & 0.324 \\
13 & 25 & 10 & 0.09 & 0.49 & 0.24 \\
11 & 21 & 9 & 0 & 0.38 & 0.197 \\
10 & 21 & 11 & 0.122 & 0.39 & 0.331 \\
12 & 23 & 15 & 0 & 0.4 & 0.25 \\
10 & 28 & 17 & 0.14 & 0.57 & 0.33 \\
\hline
\end{tabular}

(1) No. of $D_{T n}$, (2) no. of PF, (3) $R_{T n}$ assigned. 
Table 3. Comparison of GARA-SDP and HRAA in terms of average expected value of uncompleted data transmission.

\begin{tabular}{lllcccc}
\hline$(1)$ & $(2)$ & $(3)$ & $(4) \theta\left(D_{T n}\right)(\%)$ & $(4) O\left(D_{T n}\right)(\%)$ & $(5) \theta\left(D_{T n}\right)(\%)$ & 4.80 \\
\hline 22 & 25 & 21 & 2.01 & 9.34 & 5.86 & 13.18 \\
23 & 21 & 17 & 2.32 & 7.32 & 10.33 & 23.19 \\
26 & 21 & 18 & 1.43 & 9.11 & 12.96 & 13.45 \\
27 & 23 & 19 & 2.37 & 10.77 & 14.31 & 30.45 \\
24 & 28 & 17 & 1.89 & 12.35 & $\left(D_{T n}\right)$ \\
\hline
\end{tabular}

(1) No. of $D_{T n}$, (2) no. of PF, (3) $R_{T n}$ assigned, (4) GARA-SDP, (5) HRAA.

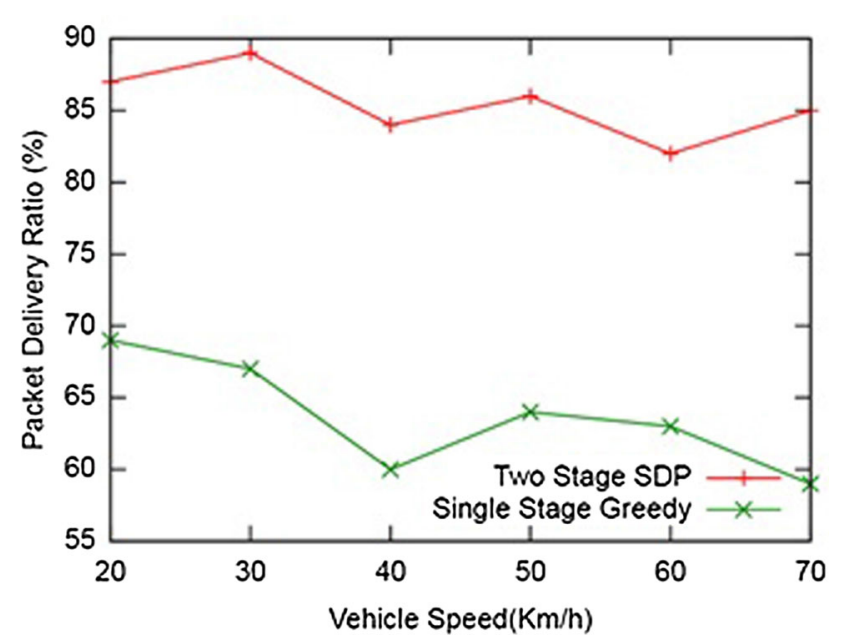

Figure 4. Packet delivery ratio vs speed of the vehicles in optimal zone.

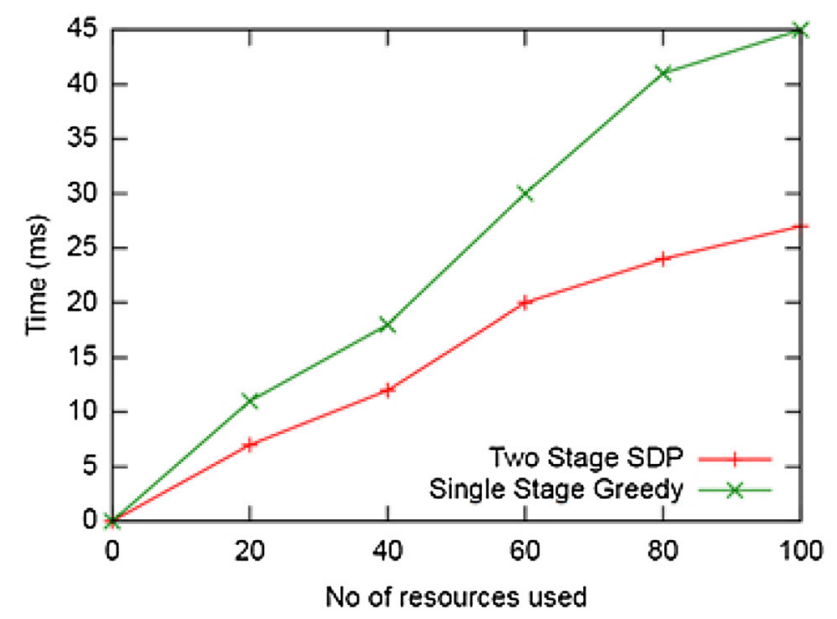

Figure 5. Routing latency vs number of resources used.

geographical-angular-zone-based two-phase resource allocation system, which uses only the dominant backbone resources for data transmission. It also considers the first phase resource allocation results before making decisions on second phase resource allocation.
The next set of simulations considered the resource allocation problem by having 20-30 data transmissions. This experiment uses the number of resources in the range of 17-22. For these problems we have examined the worst and average percentage of GARA-SDP and HRAA over 100 instances. Table 3 shows the results that confirm the optimal performance of GARA-SDP approach. The proposed approach is numerically different from IDP by 5\% of the value of average data transmission whereas HRAA algorithm has more deviation over GARA-SDP that ranges from $8 \%$ to $25 \%$.

\subsection{Packet delivery ratio}

Through this simulation set-up, we evaluated the efficiency of two-phase resource allocation by measuring the packet delivery ratio (PDR) at different speeds of the vehicles; 10 vehicles were randomly selected to send packets over single-phase resources' utilization. The same communication has been made through two-phase resource allocation environment. The simulation time for each run is $1000 \mathrm{~s}$. The vehicles speed is varied in this scenario from 20 to $70 \mathrm{~km} / \mathrm{h}$. Figure 4 illustrates that the two stages SDP attains $87 \%$ packet delivery ratio when the vehicle speed is $30 \mathrm{~km} /$ $\mathrm{h}$ and it continuously retains a higher PDR of approximately $83 \%$ when the vehicles' speed is $70 \mathrm{~km} / \mathrm{h}$. The two stages SDP showed efficient PF even though the network topology was varying over the time. The reason behind the optimal performance is that the results of all the resources assigned in the first stage are analysed before selecting the resources for PF in the second stage. The proposed two stages SDP scheme assigns higher priority to the data transmissions performed in the optimal zone. The $86 \%$ confidence interval for mean packet delivery ratio for speed of the vehicles varying from 20 to $70 \mathrm{~km} / \mathrm{h}$ is plotted in figure 4.

\subsection{Routing latency}

Figure 5 shows the routing latency of single-stage resource allocation over two-stage SDP when the number of vehicles is varied. Routing latency is found by the time taken for delivering packets to a destination through the determined 
efficient route. It is observed that the performance of the proposed scheme is more efficient over single-stage greedy resource allocation approach. When the simulation scenario has 60 resources, two stages SDP completed 100 packets transmission with $20 \mathrm{~ms}$ of routing latency whereas the time taken by the single-stage greedy was $30 \mathrm{~ms}$. The reason behind this routing latency optimization result is that the proposed work has not considered all resource count around $360^{\circ}$. It considers the resources placed only in the optimal zone (from $20^{\circ}$ to $60^{\circ}$ ). When we have allocated 80 and 100 resources for packet forwarding, it is noticed that $90 \%$ confidence interval for mean routing latency is achieved.

\subsection{Propagation delay}

The propagation delay of the proposed work was compared with those of HRAA, IDP and single-stage greedy resource allocation schemes. Formally, the propagation delay is defined as the magnitude of time taken by the code to run in all nodes in the network. All schemes' propagation delays increase when the size of the code increases. Figure 6 shows that the optimal allocation of resources by two stages SDP holds less propagation delay over existing schemes; this is because the code execution takes place only in the nodes that lie in the optimal zone that connects source and destination. Moreover, we have used the thread pool concept to write the code for resource allocation. It allows the code to execute in a parallel manner. The python bindings also provide support for multi-threading.

\subsection{Task completion hit rate}

Figure 7 shows the hit rate of the task completion when the number of resources is varied from 25 to 100 for

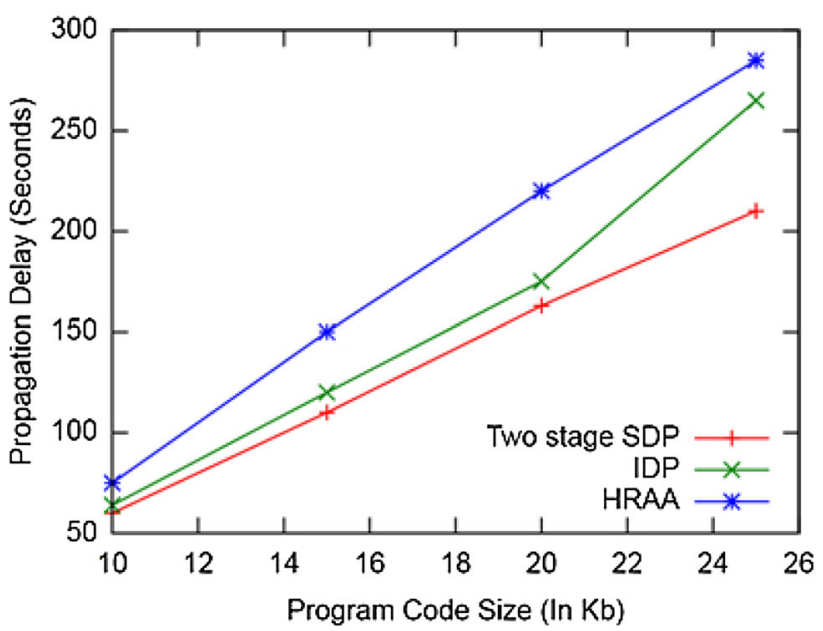

Figure 6. Propagation delay vs program code size.

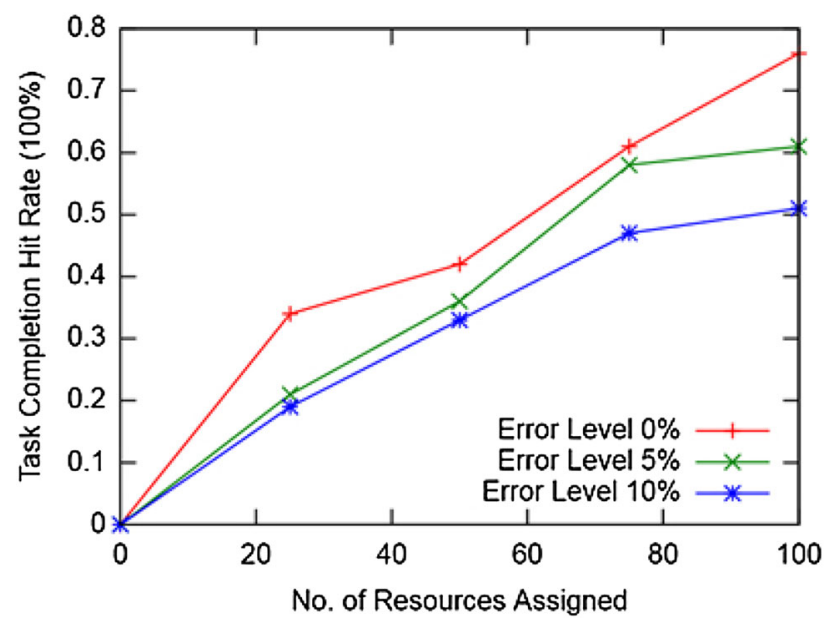

Figure 7. Task completion hit rate vs no. of resources allocated.

transmitting 200 packets of size 1024 bytes each. The same simulation has been performed in 10 rounds with various noise levels; successful completion of tasks has been observed from the two phases of resource allocation by its routing state vectors. At error level $0 \%$, the hit rate of task completion in two phases SDP resource allocation starts from $34 \%$ when 25 resources participated in the routing process of 200 packets. Later, it fluctuated between $42 \%$ and $79 \%$. At this error level, the resources have been utilized optimally and there is linear correlation with number of successful data transmissions.

\section{Conclusion and future directions}

Relaxed approximation SDP model has been used to divulge the best outcomes of individual resources' strategy over two phases to maximize the utilization of resources in VANET. The problem of unreliable resources' allocation to PF over two stages is relevant in many essential applications. The control overhead of the network is reduced by considering that limited resources lie only within selected transmission angles. This angular zone selection scheme overcomes the flooding of control packets to all nodes placed in $360^{\circ}$. The proposed SDP produces a routing state vector that records the opportunity for observing successful data transmission using multistage recourse allocation strategies. Simulation results show that the relaxed approximation SDP model is better than singlestage greedy approach, HRAA and IDP. To the best of our knowledge from the benchmark survey, this is the first work that uses the SDP for enhancing efficient routing by allocating resources in an optimal manner for VANETs. This work can be extended in several important directions. The first of these extensions is considering heterogeneous resources. The second of these is expansion of the results to multiple phases. 


\section{References}

[1] Castanon D A and Wohletz J M 2009 Model predictive control for stochastic resource allocation. IEEE Trans. Autom. Control 54(8): 1739-1750

[2] Puterman M L 2005 Markov decision processes: discrete stochastic dynamic programming. New York: John Wiley and Sons

[3] Fricker J D and Whitford R K 2004 Fundamentals of transportation engineering: a multimodal systems approach. Englewood Cliffs: Prentice Hall

[4] Rawat D B, Song M and Shetty S 2015 Resource allocation for cognitive radio enabled vehicular network users. In: Dynamic Spectrum Access for Wireless Networks. New York: Springer, pp. 57-65. ISBN: 978-3-319-15299-8

[5] Bejaoui T 2014 QoS-oriented high dynamic resource allocation in vehicular communication networks. Sci. World J. 2014: 718698

[6] Xing W, Wang N, Wang C, Liu F and Ji Y 2014 Resource allocation schemes for D2D communication used in VANETs. In: Proceedings of the 80th IEEE Vehicular Technology Conference, Vancouver, pp. 1-6. https://doi.org/ 10.1109/VTCFall.2014.6966159

[7] Araniti G, Campolo C, Condoluci M, Iera A and Molinaro A 2013 LTE for vehicular networking: a survey. Commun. Mag. 51(5): 148-157

[8] Krishnamurthy V 2002 Algorithms for optimal scheduling of hidden Markov model sensors. IEEE Trans. Signal Process. 50(6): 1382-1397

[9] Chhetri A S, Morrell D and Papandreou-Suppappola A 2006 On the use of binary programming for sensor scheduling. IEEE Trans. Signal Process. 55(6): 2826-2839

[10] Miller M and Vaidya N 2005 A MAC protocol to reduce a sensor network energy consumption using a wakeup radio. IEEE Trans. Mob. Comput. 4(3): 228-242
[11] Chhetri A S, Morrell D and Papandreou-Suppappola A 2006 Sensor resource allocation for tracking using outer approximation. IEEE Trans. Signal Process. Lett. 14(3): 213-216

[12] Kaufman B, Lilleberg J and Aazhang B 2013 Spectrum sharing scheme between cellular users and ad-hoc device-todevice users. IEEE Trans. Wirel. Commun. 12(3): 1038-1049

[13] Jarupan B and Ekici E 2009 Location and delay aware cross layer communication in V2I multi-hop vehicular networks. IEEE Commun. Mag. 47(11): 112-118

[14] Williams J L, Fisher III J W and Willsky A S 2005 An approximate dynamic programming approach to a communication constrained sensor management problem. In: Proceedings of the International Conference of Information Fusion, July

[15] Lim J M Y, Chang Y C, Alias M Y and Loo J 2014 Joint optimization and threshold structure dynamic programming with enhanced priority scheme for adaptive VANET MAC. Wireless Netw. 22(3): 897-913, https://doi.org/10.1007/ s11276-015-1009-x

[16] Cheung M H, Hou F, Wong V W and Huang J 2012 DORA: dynamic optimal random access for vehicle-to-roadside communications. IEEE J. Commun. 30(4): 792-803

[17] Dhurandher S K, Misra S, Obaidat M S, Gupta M, Diwakar K and Gupta P 2010 An efficient angular routing protocol for inter-vehicular communication in vehicular ad hoc networks. IET Commun. 4(7): 826-836

[18] Paramasivan B, Bhuvaneswari M and Pitchai K M 2015 Augmented DTN based energy efficient routing protocol for vehicular ad hoc networks. In: Proceedings of the 2015 IEEE SENSORS Conference, Busan, pp. 1-4. https://doi.org/10. 1109/ICSENS.2015.7370257

[19] Vakilinia S, Qiu D and Ali M M 2014 Optimal multi-dimensional dynamic resource allocation in mobile cloud computing. EURASIP J. Wirel. Commun. Netw.https://doi. org/10.1186/1687-1499-2014-201 